\title{
MIXED AFFECTIVE STATES: A STUDY WITHIN A COMMUNITY MENTAL HEALTH TEAM WITH TREATMENT RECOMMENDATIONS
}

\author{
Jasmine Ho ${ }^{1}$, Suarabh Singh ${ }^{1}$, Mark Agius ${ }^{2,3}$ \& Rashid Zaman ${ }^{2,3}$ \\ ${ }^{1}$ University of Cambridge School of Clinical Medicine, Cambridge, UK \\ ${ }^{2}$ South Essex Partnership University Foundation NHS Trust, UK \\ ${ }^{3}$ Department of Psychiatry University of Cambridge, UK
}

\section{SUMMARY}

Introduction: Agitated Depression and Bipolar mixed states combine depressive and manic symptoms, reflecting severe forms of affective disorders with an increased suicide risk. These states have not been defined with adequate consensus and hence present a diagnostic and therapeutic challenge. In this study, we argue that both states are part of the bipolar spectrum rather than a distinct affective syndrome or a mixed anxiety-depression state.

Methods: The current literature has been reviewed and suggestions are made for a unified definition of this entity, which would be valuable in recognising this dangerous state. We have then studied 17 cases of mixed states identified from a database held by a UK community mental health team. We have studied the treatments offered and estimated the time these treatments have taken to end the mixed state and so reduce the risk of suicide.

Results: Not surprisingly, there are no clear recommendations for treatment of these mixed states. We found that The best results were achieved with a three pronged regimen including increasing/adding mood stabilisers; and/or increasing/adding antipsychotics; as well as decreasing antidepressants. Resolution was achieved in two and a half weeks.

Discussion: From this work we have deduced several general principles, which are here reviewed and hence we have been able to suggest possible treatment strategies.

Conclusion: Because of the close link between affective mixed states and suicidality, it is of great importance that patients be brought out of the mixed affective state as soon as possible, hence we believe that our findings are of importance in managing these patients. However we recommend further study on a larger sample in order to confirm our findings.

Key words: affective mixed states - bipolar disorder - mood stabilizers - antidepressants - antipsychotics

\section{INTRODUCTION}

Mixed states combine depressive and manic symptoms and are important due to an increased suicide risk. These states have not been defined with adequate consensus and hence present a diagnostic and therapeutic challenge. Different terms, including 'Mixed affective state', 'Dysphoric mania', 'Mixed Bipolar Depression' and 'Agitated Depression' have been used. This reflects the fact that different proportions of manic and depressive symptoms may co-exist, so that three different phenotypes can be described; Mixed affective episodes (when the proportion of manic and depressive symptoms is equal), Dysphoric mania (when manic symptoms are dominant), and Mixed (bipolar) depresssion (when depressive symptoms are the dominant symptoms). This is possible because both mania and depression are active processes that can occur both successively and simultaneously (Rihmer \& Gonda 2006, Agius 2007). It also reflects the fact that Agitated depression can be reformulated as an affective mixed state (Akiskal et al. 2005). For instance, (Akiskal et al. 2005) showed that a significant percentage (19.7\%) of patients diagnosed with Unipolar depression $(n=254)$ experience 'agitated depression'. There was a strong clustering of 'non-euphoric hypomanic' symptoms in those $19.7 \%$, which indicates a Bipolar spectrum link. In fact, Tavormina et al. showed that $28 \%$ of bipolar patients had agitated depression $(n=300)$ (Tavormina \& Agius 2007). It is logical to classify these states as part of the bipolar affective spectrum disorder. Indeed, these mixed states can be conceptualised as transition states between manic and depressive episodes. Further evidence that agitated depression is in fact a $\mathrm{n}$ affective mixed state is also provided by a study by Maj et al. They found that Patients with agitated major depression were more likely to receive antipsychotics during their index episode and spent a higher proportion of time in an affective episode compared with patients with nonagitated major depression. There was an association between a family history of bipolar I disorder, more frequent suicidal thoughts during the episode of depression, a longer duration of the episode of depression, and a higher affective morbidity and the presence of two manic or hypomanic symptoms during the depressive episode (Maj 2006). Hence it was concluded that the subgroup of patients with at least 2 manic or hypomanic symptoms may be suffering from a mixed affective state and hence were part of the bipolar spectrum (Maj 2006).

"Bipolar spectrum" first appeared in the psychiatric literature in a 1977 paper on a prospective follow-up of 
cyclothymic individuals (Akiskal et al. 1977). Akiskal et al. conceptualised Unipolar and Bipolar Mixed states as 'Depressive Mixed State' and a part of the Bipolar Affective Spectrum (Akiskal et al. 2005).

Mixed states represent disturbances in mood and a combination of manic and depressive symptoms. Rhimer et al. described three entities which he modelled as two different outputs from two distinct 'mood generators' (Rihmer \& Gonda 2006). Thus, they describe three phenotypes of mixed states (according to Rihmer \& Gonda 2006). These are; 1. Mixed affective episode (full mania and full major depression simultaneously according to DSM-IV), 2. Dysphoric mania (full mania according to DSM-IV and few depressive symptoms not reaching the threshold of major depression) and 3. mixed depression (DSM-IV major depressive episode + a few hypomanic symptoms not reaching the level of hypomania/mania), (question 8).

Mixed states are not uncommon in patients with bipolar disorder. A study by Bauer showed that clinically important depressive symptoms occurred in $94.1 \%$ of patients with (hypo)mania, while $70.1 \%$ of patients who were in a depressive episode had clinically significant manic symptoms (Bauer 2005). In another study, Goldberg showed that two-thirds of subjects with bipolar depressed episodes also had concomitant manic symptoms. The most frequent symptoms were distractibility, flight of ideas or racing thoughts, and psychomotor agitation. Goldberg showed that patients with any mixed features were significantly more likely to have early age at illness onset, rapid cycling in the past year, to be of the bipolar I subtype, to have a history of suicide attempts, and to have more days in the preceding year with irritability or mood elevation (Goldberg 2009).

Mixed states have grave importance clinically because patients have high suicide risk as shown by several studies (Balázs et al. 2006). Valtonen in a study of suicide attempts in different phases of Bipolar disorder (Valtonen 2008) found that compared to the other phases of the illness, the incidence of suicide attempts was 37-fold higher during combined mixed and depressive mixed states, and 18 -fold higher $(95 \%$ CI: 6.5-50.8) during major depressive phases. Thus, the incidence of suicide attempts varies remarkably between the different illness phases, and mixed and depressive phases are the phases in which there is the highest risk. Therefore, time spent in these high-risk illness phases appears to be the major determinant of overall risk for suicide attempts among BD patients. Hence, clinically, management of mixed and depressive phases appears to be may be crucial in reducing suicide risk in bipolar patients (Valtonen 2008).

Balázs et al. (2006) showed that psychomotor agitation and irritability are strong predictors of increased suicidality (Balázs et al. 2006).

There is scant literature about how to treat mixed states. There are a few principles that emerge from the literature. There is a need to discontinue antidepressants (Thuile et al. 2005). Among mood stabilisers, Divalproate has been more efficacious (Thuile et al. 2005).

There is little evidence on the use of adjuncts: not much evidence of use.

\section{METHODS}

The study included a case series of 17 patients from an anonymised Bedford Community Mental Health Team database. These patients were selected retrospecttively from clinic letters dictated by Consultant Psychiatrists with symptoms of low mood with agitation/ irritability, high mood with agitation/irritability or a cycling mood with agitation/irritability. Risk assessment of patients was also examined, as well as the involvement of a Care Co-ordinator.

Treatment response was followed up from clinic letters. Changes to medication doses or additions of other drugs were recorded. The primary end-point considered was the duration to resolution of the mixed state.

Thus, this study is an audit of ordinary practice in a single community mental health team in Bedford, England, which deals with patients of 'working age', that is of an age range between 17 and 70 , as is usual in English CMHTs. It was a study of 'ordinary practice', rather than a trial, and it needs to be understood that the concept of 'mixed affective states' had only relatively recently been introduced into the team, by a doctor with a special interest in Bipolar Disorder. Because this was a study of 'ordinary practice', no rating scales were used to identify patients who were suicidal, but patients were routinely risk assessed for suicidality during each outpatient appointment interview, as is usually the case in English CMHTs. As is a legal requirement in the UK national health service, all diagnoses are recorded according to ICD 10. Since this was an audit of routine practice, resolution of the mixed state was determined by clinical interview, by the patients reporting that the symptoms which had led to the diagnosis of the mixed affective state had resolved.

\section{RESULTS}

Although patients ranging in age between 17 and 70 could have entered the study, in practice the age range was between 24 years and 60 years. There were 8 men and 9 women. One patient was not included in the analysis because we had incomplete data.

The main results of our study were shown in table 1. One patient was not included in the analysis because not all the data was available. Reductions in antidepressants were carried out on the basis of present NICE guidelines that patients with bipolar should not be on antidepressants unless they were depressed, in order to avoid the occurance of Mixed states or rapid cycling, so there was no fixed percentage reduction, but the aim was to stop anti-depressants all together when 
appropriate. Sometimed this could not be achieved except in stages. Equally, the intention was to increase anti-psychotics and mood stabilisers within the licensed dosages. All of the patients had a previous history of suicidal ideation or actual suicide attempts or self harm attempts recorded in the notes, however in none of the cases had this previous history been directly attributed to 'mixed affective states'. Out of 17 patients, the following diagnoses were present: 16 had Bipolar Affective Disorder, of which 7 were Type 1 and 9 were Type II. Of the 9 Type II Bipolar patients, 2 of them also had a diagnosis of Borderline Personality Disorder. The remaining patient out of the 17 had Depression and Borderline Personality Disorder.

From our case series, the following characteristics of mixed states were seen:

Table 1. Definition of a mixed state from the case series Mood Disturbance AND One or more of the following

\begin{tabular}{ll}
\hline Low & Anxiety \\
High & Agitation \\
Labile & Irritability \\
& Aggressiveness \\
& Emotional lability \\
\hline
\end{tabular}

Suicidal ideation was seen in $10 / 17$ of these patients experiencing a mixed state (59\%). In a subset of 6 patients who were agitated, 5 were suicidal (83\%). There were no completed suicides.

\section{Treatment Options}

Figure 1 shows the changes to medication and the subsequent duration of resolution of the mixed state. All patients eventually recovered but with variable time courses. The changes to the medication such as adding an atypical were gradually reversed after recovery.

Table 2. Treatment response

\begin{tabular}{ccc}
\hline & $\begin{array}{c}\text { Change to } \\
\text { medication }\end{array}$ & $\begin{array}{c}\text { Duration to } \\
\text { resolution }\end{array}$ \\
\hline A & 5 \\
B & 7.2 \\
Monotherapy & $\mathrm{D}$ & 5 \\
& $\mathrm{E}$ & 8 \\
$\mathrm{~A}+\mathrm{D}$ & 10 \\
B $+\mathrm{D}$ & 5 \\
Dual therapy & $\mathrm{A}+\mathrm{B} / \mathrm{C}$ & 4 \\
& $\mathrm{~A}+\mathrm{B}+\mathrm{D}$ & 8.5 \\
Triple therapy & \multicolumn{2}{c}{2.5} \\
\hline Legend: & \multicolumn{2}{c}{$\mathrm{A}$} \\
Increasing/adding mood stabilisers & $\mathrm{B}$ \\
Increasing/adding atypical antipsychotics & $\mathrm{C}$ \\
Increasing/adding typical antipsychotics & $\mathrm{D}$ \\
Decreasing antidepressants & $\mathrm{E}$
\end{tabular}

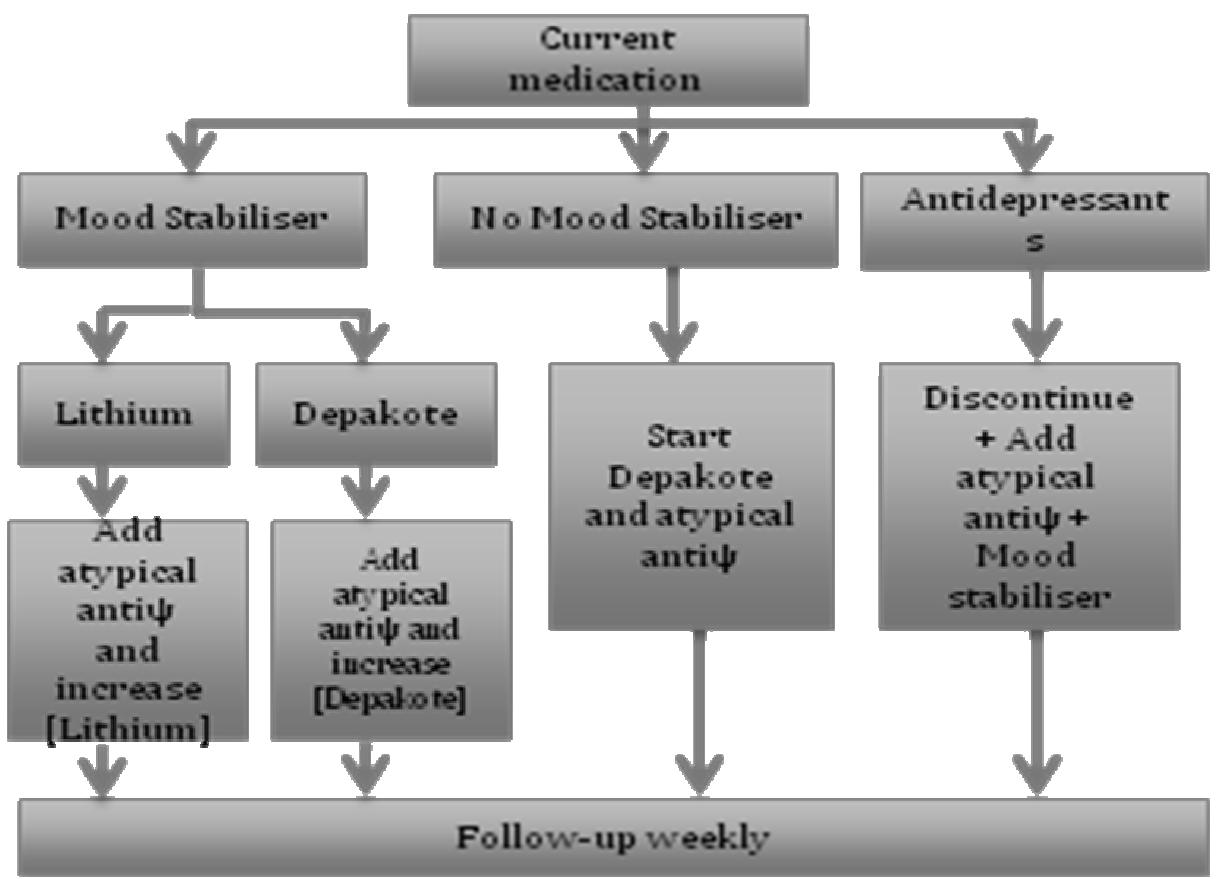

Figure 1. Management algorithm of mixed states

\section{DISCUSSION}

The following limitations were taken into account when drawing conclusions from the case series. The number of patients included in this study is relatively small and so it serves as a pilot for further research into treating mixed states. Most patients in our study were followed up weekly, however there were a few patients who were seen after longer periods. It is important to follow up patients experiencing mixed states weekly because the variability in mood could lead to risky behaviour, especially suicide. 
The presence of such a mixed state in a patient demonstrates that they are suffering from bipolar affective disorder. Therefore it is important that in all patients with an affective mixed state, a longitudinal history is taken in order to screen for hypomania and manic episodes.

Although the study was small, it showed that agitation is a strong predictor of suicide risk given that 5 patients in a subset of 6 agitated patients were suicidal. This is in concordance with larger studies.

In terms of treatment, decreasing anti-depressants is crucial in resolving mixed states. In one case, antidepressants were increased in dose, which produced the worst result with a prolonged period of resolution (10 weeks). Dual therapy such as decreasing antidepressants and Increasing/adding mood stabilisers resulted in an average period of resolution of five weeks. Slightly better results were achieved by decreasing antidepressants and increasing/adding atypical antipsychotics; giving a duration of four weeks. However, if antidepressants are not decreased, it takes longer for resolution (8.5 weeks) from increasing/adding atypical antipsychotics and mood stabilisers.

The best results were achieved with a three pronged regimen including increasing/adding mood stabilisers; and/or increasing/adding antipsychotics; as well as decreasing antidepressants. Resolution was achieved in two and a half weeks.

The ensuing algorithm summaries our findings and recommendations on treatment of mixed states:

\section{CONCLUSION}

Because of the close link between affective mixed states and suicidality, it is of great importance that patients be brought out of the mixed affective state as soon as possible, hence we believe that our findings are of importance in managing these patients. However we recommend further study on a larger sample in order to confirm our findings.

\section{REFERENCES}

1. Agius M: The Consequences of the Model of the Bipolar Spectrum for the Choice of Treatment for Bipolar Illness. Psychiatrie 2007; 11(Suppl 4):8-12.

2. Akiskal HS, Djenderedjian AM, Rosenthal RH, Khani MK: Cyclothymic disorder: validating criteria for inclusion in the bipolar affective group.Am J Psychiatry. 1977; 134(11):1227-33.

3. Akiskal HS, Benazzi F, Perugi G, Rihmer Z: Agitated "unipolar" depression re-conceptualized as a depressive mixed state: implications for the antidepressant-suicide controversy. J Affect Disord. 2005; 85:245-58.

4. Balázs J, Benazzi F, Rihmer Z, Rihmer A, Akiskal KK, Akiskal HS: The close link between suicide attempts and mixed (bipolar) depression: implications for suicide prevention. J Affect Disord. 2006; 91:133-8.

5. Bauer MS, Simon GE, Ludman E, Unützer J: 'Bipolarity' in bipolar disorder: distribution of manic and depressive symptoms in a treated population. Br J Psychiatry. 2005; 187:87-8.

6. Goldberg JF, Perlis RH, Bowden CL, Thase ME, Miklowitz DJ, Marangell LB, Calabrese JR, Nierenberg AA, Sachs GS.Manic symptoms during depressive episodes in 1,380 patients with bipolar disorder: findings from the STEP-BD.Am J Psychiatry. 2009; 166:173-81.

7. Maj M, Pirozzi R, Magliano L, Fiorillo A, Bartoli L: Agitated "unipolar" major depression: prevalence, phenomenology, and outcome. J Clin Psychiatry. 2006 May; 67(5):712-9.

8. Rihmer Z, Gonda X: Prediction and prevention of suicide in bipolar illness. The second dual congress on Psychiatry and the Neurosciences, 1st European Congress of the International Neuropsychiatric Association; 2nd Mediterranean congress of the World Federation of Societies of Biological Psychiatry Athens 2006, Book of Abstracts $p 30$.

9. Tavormina G, Agius M: A study of the incidence of bipolar spectrum disorders in a private psychiatric practice. PsychiatrDanub. 2007; 19:370-4.

10. Thuile J, Even C, Guelfi JD: Mixed states in bipolar disorders: a review of current therapeutic strategies. Encephale 2005; 31(5 Pt 1):617-23.

11. Valtonen HM, Suominen $K$, Haukka J, Mantere $O$, Leppämäki S, Arvilommi P, Isometsä ET: Differences in incidence of suicide attempts during phases of bipolar I and II disorders. Bipolar Disord. 2008; 10:588-96. 